\title{
The Neuronal Endomembrane System
}

\section{Direct Links between Rough Endoplasmic Reticulum and the cis Element of the Golgi Apparatus ${ }^{1}$}

\author{
JAMES D. LINDSEY ${ }^{2}$ AND MARK H. ELLISMAN ${ }^{3}$ \\ Department of Neurosciences, University of California, San Diego, School of Medicine, La Jolla, California 92093
}

\begin{abstract}
This is the first of three papers describing new components and structural relationships within the neuronal endomembrane system. This system includes: the nuclear envelope, rough endoplasmic reticulum, the Golgi apparatus, Iysosomes, axoplasmic reticulum, and discrete cytoplasmic compartments such as vesicles, multivesicular bodies, and so on. Previous high voltage electron microscope studies of osmium-impregnated Golgi apparatus have shown that small varicose tubules often arise from the cis element. In bullfrog spinal ganglia, these tubules have been seen to extend into cytoplasmic domains occupied by rough endoplasmic reticulum (RER). This study was undertaken to determine whether varicose tubules form direct connections with RER and to measure cytoplasmic density of varicose tubules. Spinal ganglia from Rana catesbeiana were fixed by osmium impregnation and, in some cases, were then stained en bloc with lead aspartate. Sixty- to $\mathbf{3 0 0 0 - n m - t h i c k ~ s e c t i o n s ~ o f ~ t h i s ~ t i s - ~}$ sue, as well as of ganglia prepared by conventional protocol, were studied using standard and high voltage electron microscopy. Quantitative analysis revealed that $9 \%$ of the varicose tubules connected with the Golgi cis element. To determine whether continuities existed between RER and any of these tubules, more than $\mathbf{5 0}$ tubules were fully reconstructed from images of serial sections. The "ends" of these reconstructed tubules were then studied by high magnification stereoscopy. This was facilitated by the en bloc lead staining which allowed visualization of unimpregnated RER elements along with the impregnated tubules. Small varicose tubules as well as larger smooth tubules were found to have
\end{abstract}

Received May 14, 1984; Revised May 16, 1985;

Accepted May 23, 1985

This work was supported by grants from the National Institutes of Health (NS14718), the Muscular Dystrophy Association, the National Multiple Sclerosis Society; and the Epilepsy Foundation of America to M. H. E. J. D. L. was supported by a National Science Foundation predoctoral fellowship during a portion of this work. We wish to thank Dolores Taitano for word processing. Thomas Deerinck and Derek Leong for technical assistance, Dr. Stephen Young for valuable assistance during the computer reconstructions, Dr. Philip Groves for use of his computing facilities, and the University of Colorado for use of the National High Voltage Electron Micro. scope Resource Facility (P41-RR00592).

${ }^{2}$ Current address: Retinal Degeneration Center, Wilmer Ophthalmological Institute, Johns Hopkins University School of Medicine, 600 North Wolfe Street, Baltimore, MD 21205.

${ }^{3}$ To whom correspondence should be addressed. formed confluent bridges between the cis element and RER. In addition, the varicose tubules were found to bridge widely separated elements of RER. Finally, numerous examples of varicose and smooth tubules were seen to extend from RER and from cis elements to eventually form blind endings. These findings raise the possibility that the tubules form highly dynamic transitory connections between RER and the Golgi apparatus as well as between separated elements of RER. Those between RER and Golgi apparatus are ideally positioned to play a major role in the transfer of protein or lipid components first assembled in RER to the Golgi apparatus for terminal synthetic modification.

This is the first in a series of three papcrs that describe new findings within the structural organization of the neuronal endomembrane system of spinal ganglion neurons. This endomembrane system includes: the nuclear envelope; the rough endoplasmic reticulum (RER); the Golgi apparatus; discrete elements such as vcsicles, multivesicular bodies, and so on; and several different types of membranous cytoplasmic tubules. These structures have been strongly implicated as the subcellular compartments within many molecules that are essential for neurotransmission and are first synthesized and then transported by fast axonal transport to their functional locations (Grafstein and Forman, 1980; Ellisman and Lindsey, 1982). Although much is known about synthetic events occurring within RER and the Golgi apparatus, comparatively little is known about how partially assembled macromolecules are transferred from the RER to the Golgi apparatus, through the stack of cisternae that constitute the Golgi apparatus, or from the Golgi apparatus to the membranous compartments that are likely to carry Golgi apparatus products to their functional sites within neuronal processes. These papers provide new morphological evidence on the structure and relationship of endomembrane components that address all three of these issues. The present paper described a group of membranous tubules that may be involved in transporting cell products between RER and the Golgi apparatus. The second paper (Lindsey and Ellisman, 1985a) describes a variation in the three-dimensional architecture of the Golgi apparatus cis element that may be cyclical in nature. The third paper (Lindsey and Ellisman, $1985 \mathrm{~b}$ ) addresses the origin of and the relationship between discrete membranous elements found within the axoplasm and the axoplasmic reticulum.

In many cell types, including neurons, it is well established that most cell surface or secretory proteins are assembled first in RER and subsequently travel through the Golgi apparatus before reaching their final destination. This was originally confirmed in neurons by 
autoradiography (Droz, 1967; Droz and Koenig, 1970). Using a pharmacological approach, it was confirmed for spinal ganglion neurons in particular (Hammerschlag et al., 1982). This transfer of RER products to the Golgi apparatus has recently been shown in Chinese hamster ovary cells to be irilially to just one side of the Golgi stack (Bergmann and Singer, 1983). This is followed by a rapid spread of transferred material through the Golgi stack. Although the metabolic requirements of this event have been known for well over a decade (see Jamieson and Palade, 1968a, b), the anatomical mechanism of this transfer step remains highly uncertain. Furthermore, recent studies also suggest that, in addition to the orthograde transfer of cell products from RER to the Golgi apparatus, there may be a selective retrograde transfer of material from the Golgi apparatus to RER (Rothman, 1982). If retrograde transfer is supported by further studies, then the transfer machinery between RER and the Golgi apparatus is likely to prove quite sophisticated. In light of this it becomes of major importance to obtain a thorough understanding of the ultrastructural relationships between RER and the Golgi apparatus.

Dircct mombranous connections between RER and the Golgi apparatus have been reported by several authors (Novikoff et al., 1966; Flickinger, 1969; Claude, 1970; Maul and Brinkley, 1970; Holtzman, 1971; Franke and Kartenbeck, 1976; Broadwell and Cataldo, 1983). These exceptions aside, such connections are usually not found in conventionally prepared thin-sectioned material (reviewed in Morré and Ovtracht, 1977; Holtzman and Mercurio, 1980; Morré, 1981; Farquhar and Palade, 1981). This may account for the current popularity of the concept advanced by Palade (1975) that RER products move to the Golgi apparatus via discrete transition vesicles or compartments. This latter concept is based on numerous observations in many different tissues of vesicular profiles filling the space between RER and the cis element of the Golgi apparatus. However, based on kinetic considerations of protein movement from RER to the Golgi apparatus, some reservations remain about the plausibility of the transition vesicle concept (Tartakoff, 1980).

A possible resolution of this controversy comes from several studies that utilized the high voltage electron microscope (HVEM). When thick sections of osmium-impregnated rat spinal ganglion neurons were examined with the HVEM, small impregnated tubules were seen in the cytoplasm that occasionally were observed to connect directly to the edges of the cis saccule (Rambourg and Chrétien, 1970; Rambourg et al., 1974). Also impregnated were clusters of vesicles or tubules seen nearby the cis saccules. In a study of rat trigeminal ganglion neurons, it was noted that the cytoplasmic tubules could be sorted into two groups based on their appearance (Rambourg et al., 1973). The first group was narrower than the second and exhibited a regularly varicose caliber. The wider group, in contrast, was generally of smooth or constant caliber. Inferra and Carrozza (1975) noted that, in addition to the dense, confluent staining of the Golig cis face and its associated tubules, osmium impregnation sometimes results in light granular deposits within the RER. When thick sections of impregnated bullfrog spinal ganglion neurons were examined recently with the HVEM, varicuse tubules were seen to arise from both the edges and the body of cis saccules and extended into cytoplasmic regions occupied by RER (Ellisman and Lindsey, 1982). Although this raises the possibility of a confluent link between these tubules and RER, more direct evidence is needed to support this hypothesis. Thus, the present study was undertaken to resolve the following specific issues (1) What are the various types of osmophilic tubules and how frequently do they appear in the cytoplasm? (2) Is the connection of these tubules to the cis element a rare or common occurrence? (3) What is the intimate relation of varicose and smooth tubules with RER?

The results of these experiments have previously appeared in abstract form (Lindsey and Ellisman, 1983).

\section{Materials and Methods}

Tissue preparation and microscopy. Spinal ganglia from Rana catesbeiana were fixed by immersion in $2 \%$ aqueous osmium tetroxide for $1 \mathrm{hr}$. Fixation temperatures of $0,4,22$, and $37^{\circ} \mathrm{C}$ were tested to optimize preservation and efficiency of impregnation. All material analyzed in this study is from ganglia initially fixed at $4^{\circ} \mathrm{C}$. Tissues fixed at $4^{\circ} \mathrm{C}$ were best preserved, although preservation at all temperatures were adequate. Subsequently the tissue was impregnated in the same solution for 35 to $44 \mathrm{hr}$ at $37^{\circ} \mathrm{C}$ with one change of the solution at $16 \mathrm{hr}$. Length of this second incubation influenced the extensiveness of the impregnation, with $36 \mathrm{hr}$ being optimal. Following the impregnation, the ganglia wore bricfly washod in distilled water and some cases were subsequently stained en bloc with lead aspartate (Walton, 1977) for $1 \mathrm{hr}$. Other spinal ganglia were initially fixed in $1.0 \%$ glutaraldehyde, $1.0 \%$ paraformaldehyde in $0.12 \mathrm{M}$ cacodylale buffer, rinsed in buffer, and then postfixed in buffered $1 \% \mathrm{OsO}_{4}$ for $1 \mathrm{hr}$. All ganglia were dehydrated in a graded ethanol series and then gradually transferred into acetone before embeddıng in Epon/Araldite.

Thin sections were grid stained with uranyl acetate and lead citrate before examination at $80 \mathrm{KeV}$ on a JEM $100 \mathrm{CX}$ microscope equipped with a goniometric stage. Thicker sections up to $250 \mathrm{~nm}$ thick were sometimes grid stained and always carbon stabilized before examination at $100 \mathrm{KeV}$ with the instrument mentioned above. Thick sections $(1.0,2.0$, and $3.0 \mu \mathrm{m})$ were examined at $1 \mathrm{MeV}$ with the JEM 1000 HVEM at Boulder, Colorado. Thicknesses of sections thinner than $200 \mathrm{~nm}$ were estimated based on interference color reflections.

Analysis of serial sections to determine definitively whether connections between organelles exist. Serial sections (120 and $170 \mathrm{~nm}$ thick) from tissue stained en bloc with Walton's lead aspartate (Walton, 1977) were collected on Formvar-coated slot grids, carbon stabilized, and examined at $100 \mathrm{KeV}$. Initially, Golgi apparatus images were collected at low magnification (instrument magnification $\times 6600)$ and enlarged to $20 \times 25 \mathrm{~cm}$ prints. These prints were reproduced onto xerographic transparencies (Chartpak, AFX25) using a Minolta EP53OR copier at exactly 100\% original mangnification. (Most photocopiers were found not to reproduce at exactly $100 \%$.) These film reproductions were then overlaid onto prints from adjacent sections to facilitate mapping the course of the small varicose tubules as they traveled from section to section. Based on these maps, high magnification images of the specimen at various tilts for stereoscopy were obtained from the regions of sections where the small varicose tubules appeared to end and from the corresponding regions of the two adjacent sections. These images were then viewed in stereo to determine whether a continuity of the tubule end existed with any other structure.

Computer-aided serial reconstruction drawings of the Golgi apparatus and the proximal portion of small varicose tubules as they anastomosed with the cis saccule or marginal tubules were made to determine the spatial interrelationships of these structures. Alignment marks were made at four separate points on the first xerographic film image from a series using a felt-tipped marking pen. On a light box, the next film image of the series was aligned with the first based on ultrastructural landmarks such as lysosomes and mitochondria. The alignment marks on the first film image were then duplicated on the second film image with the felt pen. This was repeated in sequence for all of the film images in a given set. Using the alignment marks to position the images on a Summagraphics SPG digitizing tablet, the Golgi apparatus profiles were then traced into a Digital Equipment Corp. PDP 11 . 03 computer. This computer, using a program to suppress hidden lines, generated reconstructed drawings of the Golgi apparatus using a Tektronics 4662 interactive digital plotter.

Quantitative analysis of HVEM images to determine volumetric density of varicose tubules. Stereo irrages of $2-\mu \mathrm{rr}$-thick sections were analyzed to determine the volumetric density of the various types of small varicose tubules in cytoplasmic space. For this analysis the tubules were scored as falling into one of the following categories: (1) arising from the cis saccules and ending within the section; (2) arising from the cis saccule and abutting either the upper and lower surface of the section (presumably leaving the section before ending); (3) arising and ending within the cytoplasm of the section; (4) arising within the cytoplasm and abutting either surface of the section; and (5) abutting the surface(s) of the section on both ends of the visible segment (presumably passing into and then out of the section). A grid calibrated to the magnification of the micrograph to delineate $1.0-\mu \mathrm{m}$ squares was laid over the $0^{\circ}$ tilt micrograph of each serics, and the number of intersections over cytoplasm was counted. These scores were used to calculate, taking into consideration the $2.0-\mu \mathrm{m}$ thickness of the sections, the volume of cytoplasm analyzed. These volume scores were then used to calculate the volumetric density of the various types of varicose tubules.

\section{Results}

\section{General morphology of the osmium-impregnated ganglia}

The cytological preservation and reproducibility of the osmium impregnation. The strength and validity of our detailed observations 
are dependent upon the quality of the cytological preservation and the reproducibility of the extent of impregnation. In experiments varying parameters of the osmium impregnation procedure, it was found that the quality of preservation is largely determined by the initial fixation temperature. Changing the duration of the impregnation phase mainly influenced the amount of osmium deposited. To assess the quality of preservation, thin section images from impregnated ganglion cells were compared to ones obtained from glutaraldehyde/paraformaldehyde-fixed ganglion cells. The appearance of the endomembrane components within osmium-impregnated spinal ganglia (Fig. 1a) was found to be nearly equivalent to their appearance within thin sections of conventionally prepared ganglia (Fig. $1 b$ ). In particular, the shape of Golgi cisternae and other membranous organelles in impregnated tissue appeared very similar in both proparations. Some structures that were not as easily visualized in osmium-fixed material included microtubules and the microtrabecular lattice.

The intensity of membrane system impregnation within the neuronal soma was generally uniform (see Fig. 2). The impregnation intensity, however, varies to a minor degree within a given preparation from cell to cell. By careful comparison, it was seen that a rigid hierarchy of relative impregnability exists among the various endomembrane components. The impregnation of different structures always adhered to a specific hierarchy. Thus is it possible to gauge the intensity of impregnation. Meaningful comparisons could be made between cells exhibiting similar levels of impregnation.

\section{Osmiophilic tubule systems of the neuronal soma}

All cells studied in $2-\mu \mathrm{m}$-thick sections viewed with the HVEM were restricted to those that exhibited light to negligible granular staining of RER. The varicose tubules observed could be separated into two types. The first type extended from the cis element into the cytoplasmic domains occupied by the RER. These tubules terminated without connecting directly to other impregnated membranous organelles (Figs. 3 and 4). The endings were sometimes accompanied by a cluster of small, rounded or elongated cisternae. The second type of varicose tubule appeared both to start and end without connecting to any other impregnated structures (see Fig. 4). A quantitative analysis of 453 different varicose tubules from cytoplasmic fields of 10 different $2 \mu \mathrm{m}$ thick HVEM stcrcopair micro graphs revealed $9 \%$ to be of the cis element-connected type, whereas $91 \%$ were non-cis element connected. (Table I). Sometimes small clusters of vesicles were seen near one end of the non-cis element-connected varicose tubules. This was seen in both cis element-connected types and non-cis element-connected types. However, small clusters of vesicles were often also seen without a varicose tubule nearby (Figs. 3 and 4). Smooth tubules were much less common than varicose tubules and could usually be found to connect to a Golgi cis element (Figs. 3 and 4).

The frequency with which tubules arise from the cis element was assessed by analyzing serial section images of heavily impregnated ganglion cells (such as those shown in Figs. 6, 7, 8, and 10). Images from 170-nm-thick sections were used for this part of the analysis as it was easier to detect the presence of tubules very close to the surface of the cis element than in the HVEM-derived images of thick sections. These images were entered into a computer by tracing the Golgi stack profiles on micrographs taped to the digitizing tablet. Once a series of sections was entered, they were assembled into a reconstruction and could be displayed using a program capable of suppressing hidden lines. These reconstructions were rotated within the working buffer of the computer to facilitate examination of structures hidden due to the geometry of the Goigi apparatus. Paired drawings of the Golgi apparatus at two rotational positions were obtained in order to view stereoscopically the reconstruction and aid understanding of the often complicated drawings. These drawings revealed that tubules arising from the cis saccule were a relatively common occurrence (Fig. 5a). These reconstructions also show that Golgi stacks that appear adjacent in thin sections were usually part of the same structure (Fig. $5 b$ ).
Possible continuities between the varicose tubules and other unimpregnated membrane systems were assessed by serial section reconstruction analysis. Stereo pair images from serial sections were collected that included the full extent of various types of varicose or smooth tubule elements. Suspected transitions or confluency of a tubule with RER at the ends of an osmiophilic region were evaluated by stereoscopic viewing of the area of the section suspected of containing the transition at high magnification. For this analysis preparations of ganglia stained en bloc with lead aspartate, which allowed visualization of RER, were used. Cells were chosen that were more intensely impregnated than those used for the HVEM analysis described earlier. In such cells, intermediate saccules of the Golgi apparatus, in addition to cis saccules, were often impregnated (see Fig. 7a). Stereoscopy proved to be essential, as the twodimensional image sometimes suggested an anastomosis between a varicose tubule and a segment of RER when the structures were merely overlapped. In spite of this optimization, many of the tubules studied were rejected for detailed analysis because of ambiguities in their reconstruction or because they exited the cytoplasmic volume defined by the serial sections.

Two forms of Golgi cis element-connected varicose tubules were identified by reconstruction tracing varicose tubules. The first was attached to the Golgi cis element and then terminated without other apparent connections (Fig. 6, VT1). Occasionally a cluster of small discrete cisternae would lie near such "blind endings" (Fig. 6b). The second form of varicose tubule provided a continuous link between the Golgi cis element and the RER (Fig. 7). Similar variations of the larger cis element-connected smooth tubules that ended blindly (Fig. 6, ST1) and that connected with RER (Fig. 6, ST2) were seen.

Reconstructive tracing of non-Golgi cis element-connected varicose tubules also defined two additional form variations (Fig. 8). In both, at least one end was continuous with an element of RER. At the other end, one form connected with a second elemenl of RER (Fig. 9). The other end of the second form terminated in true blind endings (not shown).

\section{Osmiophilic vesicles of the neuronal soma}

In addition to varicose and smooth tubules, osmiophilic vesicles were often seen near the cis element. In $2-\mu \mathrm{m}$-thick sections examined with the HVEM, several impregnated vesicle clusters, in addition to discrete impregnated vesicles, were often seen closely apposed to the cis element (Fig. 3). In 170- $\mu$ m-thick sections, very tight clusters of impregnated vesicles were occasionally seen immediately adjacent to the cis element

\section{Discussion}

The present observations demonstrate that direct linkages between the Golgi apparatus cis element and the RER occur in spinal ganglion neurons. Numerous osmiophilic vesicles were also observed adjacent to the Golgi cis element. Since both the tubules and the vesicles are found between RER and Golgi apparatus, they could both be involved in the transport of cell products between RER and the Golgi cis element.

Previous observations indicating some form of continuity between RER cisternae and the Golgi apparatus have been reviewed recently by Farquhar and Palade (1981). Although such observations are few, their nature is interesting in the context of those detailed in this report. An apposition of membranous cisternae bearing ribosomes on one side was observed in association with one pole of Golgi stacks in normal cultured dorsal root ganglion neurons following exposure to x-ray irradiation (Holtzman, 1971). Continuities observed in the other reports appear as smooth (agranular) extensions of RER that approached and then extend over the cis surface of a Golgi stack. Observations of this type include: just-renucleated amoeba that had been enucleated for an extended period (Flickinger, 1969), hepatocytes of just-fed starving rats (Claude, 1970), and tissue from human melanoma (Maul and Brinkley, 1970), rat mammary adenocarcinoma (Franke and Kartenbeck, 1976), and rat hepatorna cells (Novikoff et al., 1966). Recently, a direct connection from RER to 

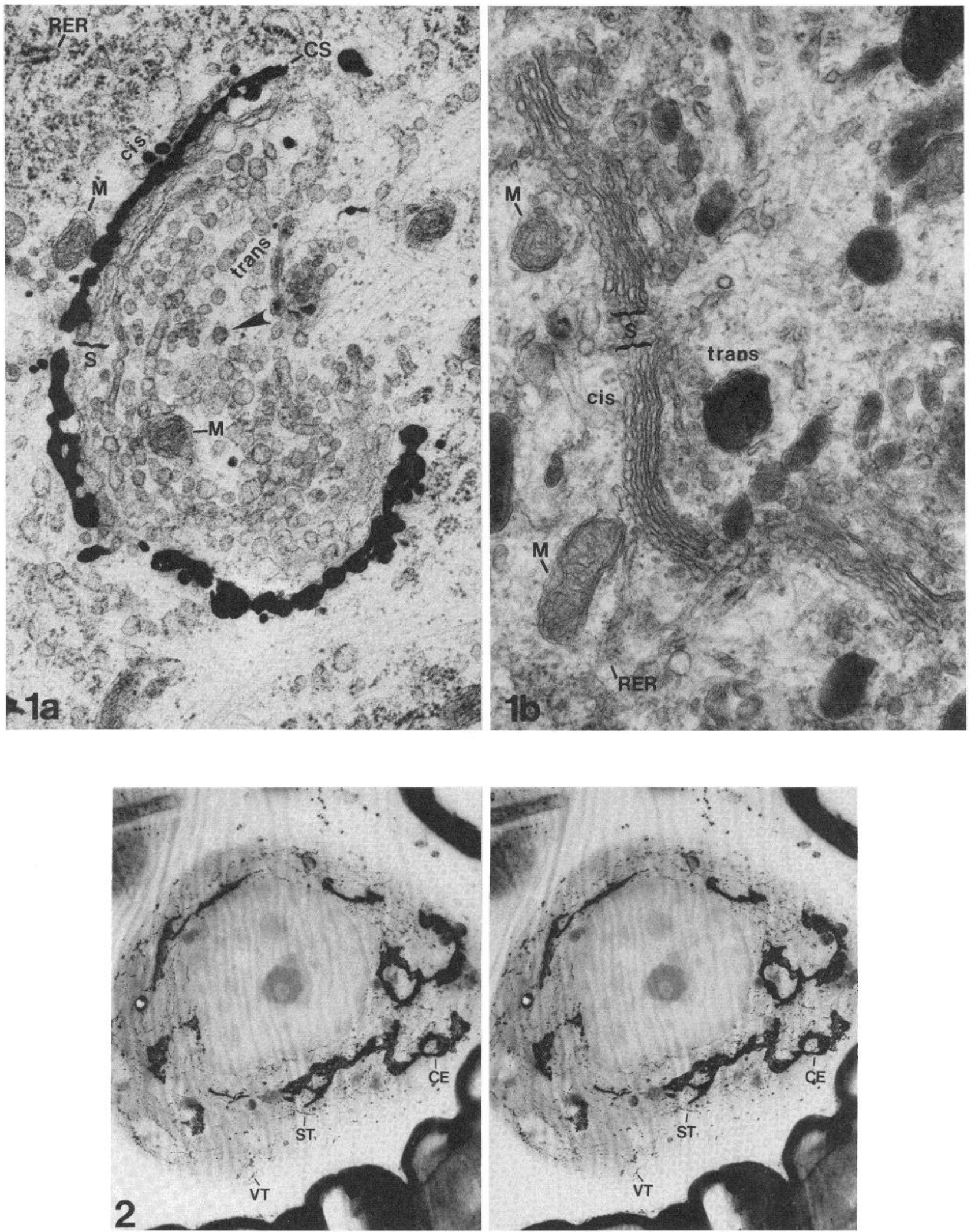

Figure 1. Golgi apparatus profiles seen in thin sections ( $90 \mathrm{~nm}$ thick) of spinal ganglia neurons prepared by osmium impregnation (a) and by conventional aldehyde-osmium fixation (b). The cis saccule (CS) of the Golgi stack is deeply impregnated following osmium impregnation. In both cases, the saccules $(S)$ are perpendicularly cross-sectioned at the top of the images and become tangentially sectioned toward the bottom of the images. The similar shapes of the saccules, of mitochondria, and their cristae $(M)$, and of rough endoplasmic reticulum membranes $(R E R)$ indicate that morphological preservation of membranous organelles is quite comparable between these two fixation techniques. The characteristic granular osmium deposits within the lumen of the RER prove to be a useful feature for the identification of this organelle system in thicker sections. Coated vesicles (arrowhead) are rarely seen. Magnification $\times 36,000$.

Figures 2 to 4 . Thick sections of osmium-impregnated spinal ganglion neurons viewed at $1 \mathrm{MeV}$ with the HVEM. In these sections, the only visible cytoplasmic structures are those that have become impregnated with osmium. Because of their thickness, however, these sections allow one to see the three-dimensional organization of the impregnated structures. These and subsequent stereo pair micrographs have been constructed for easy viewing using a $\times 2$ magnifying stereo viewer that is readily available from several of the larger electron microscope supply houses.

Figure 2. Cell body of a small spinal ganglion neuron. This and subsequent stereo pair micrographs are designed to be viewed with $\times 2$ magnifying stereo viewers readily available from several of the larger electron microscope supply houses. Note the extensive cis element (CE) of the Golgi apparatus, the ubiquitous varicose tubules $(V T)$, and the occasional smooth tubules $(S T)$ that fill the cytoplasm surrounding the cell nucleus. The section is $2 \mu \mathrm{m}$ thick, $5^{\circ}$ tilt. Magnification $\times 3,000$. 

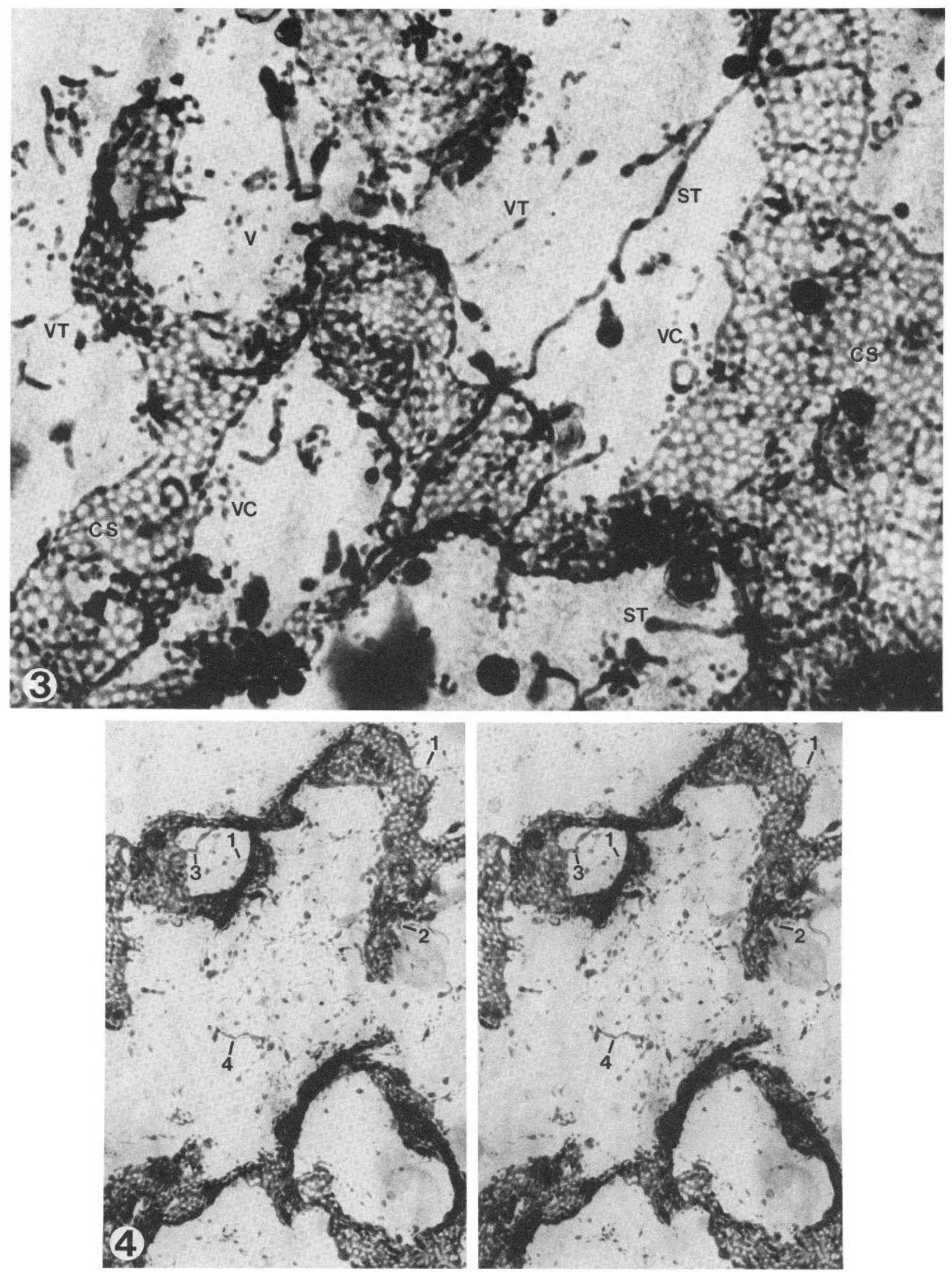

Figure 3. Higher magnification image of cis element and associated tubules (3- $\mu \mathrm{m}$-thick section). The main component of the cis element here is the sheet-like cis saccule $(C S)$. Varicose tubules $(V T)$ and smooth tubules $(S T)$ are seen to arise from the cis element. Discrete impregnated vesicles $(V)$ as well as clusters of impregnated vesicles (VC) are also seen. Magnification $\times 18,000$.

Figure 4. Stereoscopic examination allows the distinguishing of varicose tubules which pass near and are merely overlapped by the cis element (1) from those that form direct connections with the cis element (2). Smooth tubules that arise from the cis element (3) and that are unrelated to the cis element (4) are also seen. $2 \mu \mathrm{m}$ thick, $3^{\circ}$ tilt, $\times 16,000$. 


\section{TABLE I}

Mean densities and relative percentages of the various types of varicose tubules seen in 2- $\mu$ m-thick sections viewed with the HVEM

The varicose tubule type categories are: GA:cyt, varicose tubules that connect with the Golgi apparatus cis element at one end and are within the cytoplasm at the other end; GA:out, varicose tubules that connect with the cis element at one end and exit out of the section at the other end; cyt:cyt, varicose tubules that both begin and end within cytoplasm; cyt:out, varicose tubules that begin within cytoplasm and then exit out of the section; out:out, varicose tubules that pass completely through the section. Assuming random spatial distribution, only half of the varicose tubules that connect with the cis element in the adjacent sections and extends into the evaluated section to end within cytoplasm. Thus, the total percentage of varicose tubules that arose from the cis element is given by: $7.1 \%+2(1.0 \%)=9.1 \%$. The percentage that begins and ends within cytoplasm is given by: $68.0 \%+(23.6 \%-1.0 \%)$ $=90.6 \%$.

\begin{tabular}{lccccccc}
\hline \multicolumn{1}{c}{ Measurement } & GA:cyt & GA:out & cyt:cyt & cyt:out & out:out & Total & $N$ \\
\hline $\begin{array}{l}\text { Mean density } \\
\text { (number } / \mu \mathrm{m}^{3} \pm \mathrm{SEM} \text { ) }\end{array}$ & $0.023 \pm 0.006$ & $0.0033 \pm 0.0022$ & $0.23 \pm 0.05$ & $0.078 \pm 0.018$ & $0.001 \pm 0.0007$ & $0.33 \pm 0.07$ & 10 sections \\
Relative percentages & 7.1 & 1.0 & 68.0 & 23.6 & 0.3 & $(453$ tubules) \\
\hline
\end{tabular}

the cis saccules of the Golgi apparatus was reported in neurons within the nucleus of the seventh cranial nerve of mouse (Broadwell and Cataldo, 1983). A common feature in all of these examples, except in the neurons where no separate agranular bridge was reported, is that the bridge was of approximately the same width as the RER element to which the Golgi saccule was connected. Since the smooth tubule interconnections observed in the present study share this width characteristic, it may be analogous with the type of bridging elements noted in the above systems. In contrast, the varicose tubule interconnections of this report have tubular elements of a much narrower width than either the RER elements or the Golgi saccules. Thus, elements of the varicose tubule appear to constitute a previously unknown linkage between the RER and the Golgi apparatus. Two reasons that possibly contribute to their being overlooked until now are: (1) that in conventionally prepared thin sections, they usually appear as small fragments, and (2) that these fragments may look very much like segments of microtubules (see comparison in Fig. 10).

The appearance of many blind tubule endings may indicate that varicose tubules are components of a dynamic tubular network with the bridges observed here occurring transiently. Thus, caution should be exercised in modeling whether the observed densities of cis element-connected tubules presented in Table I are sufficient to account for the transfer of all RER products destined for subsequent processing in the Golgi apparatus. Interpretation of these results is, further, made even more complex by the observation that the cis element appears differently in similar-type cells from the same ganglia (see Lindsey and Ellisman, 1985a). The finding that the majority of varicose tubules are positionally related to RER alone (namely, the non-cis element-connected tubules) raises the possibility that this type of tubule may be involved in functions different from those of their cis element-connected variants. Since there is no evidence yet suggesting that the cell transfers nascent molecules among its various RER domains, any suggestion as to the function of these tubules would be highly speculative. However, due to the similarities in appearance, staining characteristics (osmium impregnability), and RER continuities, these non-cis element-connected varicose tubules may involve functions similar to those of the axoplasmic reticulum found within the axon, another membrane system for which no function is yet known (see Lindsey and Ellisman, 1985b). Alternatively, these RER-connected tubules may be either preparing to form connections with the Golgi apparatus cis element or resorbing after such a connection has been broken.

An important consideration is whether the varicose tubules are found in tissues other than adult bullfrog spinal ganglia. Presently, evidence for the presence of varicose tubuies in other cell types can be obtained by examining the figures from prevous thick- section HVEM studies of osmium-impregnated tissue. Careful examination of these figures revealed unmistakable profiles of thin varicose tubules (occasionally labeled by the authors with an arrow or a " $\mathrm{t}$ " for tubule) in all of the following rat cells: Gasserian ganglion, semilunar ganglion, trigeminal ganglion, and spinal ganglion neurons; pancreatic acinar cells; and Leydig and Sertoli cells from testes (Rambourg and Chrétien, 1970; Rambourg et al., 1973, 1974). In snail, varicose tubules are seen in the HVEM images of osmiumimpregnated multifidous gland (Carasso et al., 1971). Thus, varicose tubules appear to be ubiquitous components of animal cell architecture. Whether the varicose tubles seen in the above cell types form all of the connections reported in the present study will have to await the results of further serial section studies.

The presence of many vesicles near the cis element suggests that, in addition to varicose tubules and smooth tubules, they also play an important role in the transfer of material to or from the cis element. Recently, interest in the role of vesicular transfer has been strengthened by findings that suggest that clathrin plays a major role in the formation of vesicles from RER or the cis element (reviewed in Rothman, 1982). Because coated vesicles are only rarely seen in spinal ganglion neurons (see Fig. 1a), it is possible that the coat remains associated with the vesicle for only a very short time after vesicle formation, or that the coat is not preserved by osmium impregnation procedures, or, as a third and less likely alternative, that the role of clathrin coats is less important in this system here than in other cell types.

In conclusion, the present study demonstrates an extensive direct membranous linking of RER to the cis element of the Golgi apparatus via varicose and, on occasion, smooth tubules. Since these tubules are seen in a wide variety of animal cell types, it is likely that these tubules in general play a major role in the transport of cell products between RER and the Golgi apparatus.

Most easily impregnated is the cis saccule itself, a system of wide parallel tubules that sometimes appeared closely apposed to the cis saccule, a collection of small clustered as well as discrete vesicles that appeared close to the cis pole, and the smooth and varicose tubules. At the next more intense impregnation level, the RER exhibits a light granular staining without any change in the impregnation of other sturctures in the cell. By adjusting the impregnation conditions, most cells will have the cis element and associated structures consistently impregnated with moderately variable, light granular, RER staining. At the next level, the RER takes on a heavy granular appearance and the intermediate saccules of the Golgi apparatus become impregnated (see Fig. 6a). No example in which the intermediate saccules were impregnated was found in which the RER had not taken on this characteristic heavy granular appearance. Cells impregnated this intensely were unsuitable for HVEM analysis of thick sections because of the heightened background "noise" due to this granular RER staining. Finally, the last structure to become fully impregnated is the RER. This occurs following prolonged impregnation at $37^{\circ} \mathrm{C}$. The trans saccule of the Golgi stack and the vesicles which accumulate near the trans face were never seen to be collectively impregnated. On rare occasions, isolated impregnated vesicles were observed near the trans face. This last impregnation level is not useful for study of sections thicker than roughly 


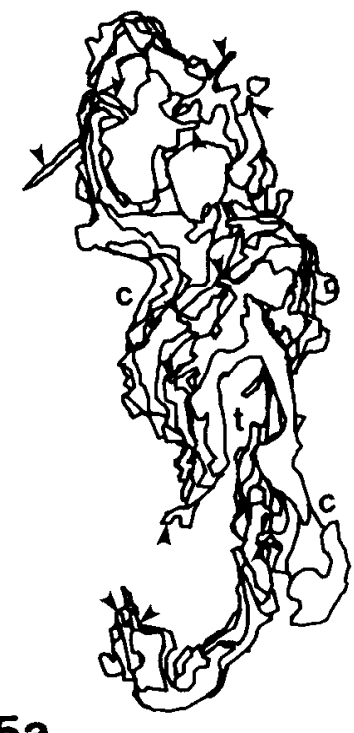

$5 a$

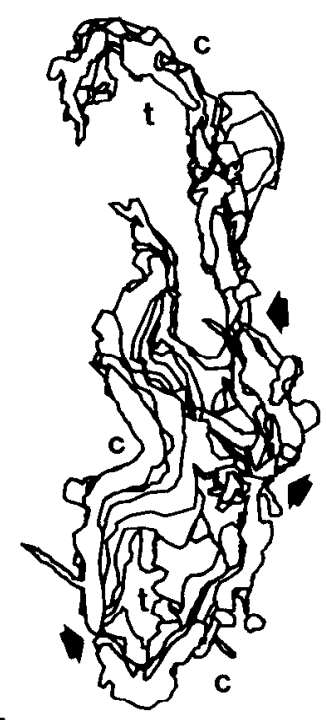

$5 b$
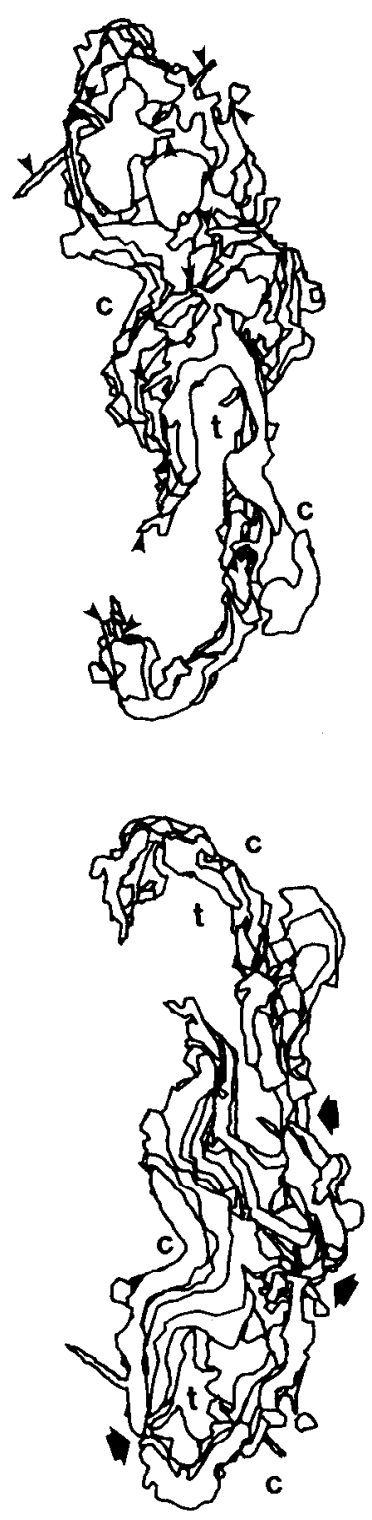

rigure 5. Stereoscopic computer reconstruction of an impregnated Golgi apparatus from a large neuron as it appeared in nine serial 170 -nm-thick sections. The reconstruction is viewed from opposite ends in $a$ and b. a favors viewing of the cis (c) surface of the Golgi stack, whereas $b$ favors viewing of the trans $(t)$ surface. The proximal portions of the relatively common varicose and smooth tubules that arise from the cis element are shown in their respestive planes of origin (arrowheads in a). b illustrates that Golgi stacks appearing adjacent in thin sections can be part of the same Golgi saccule. Scale magnification: $\times 10,000$. 

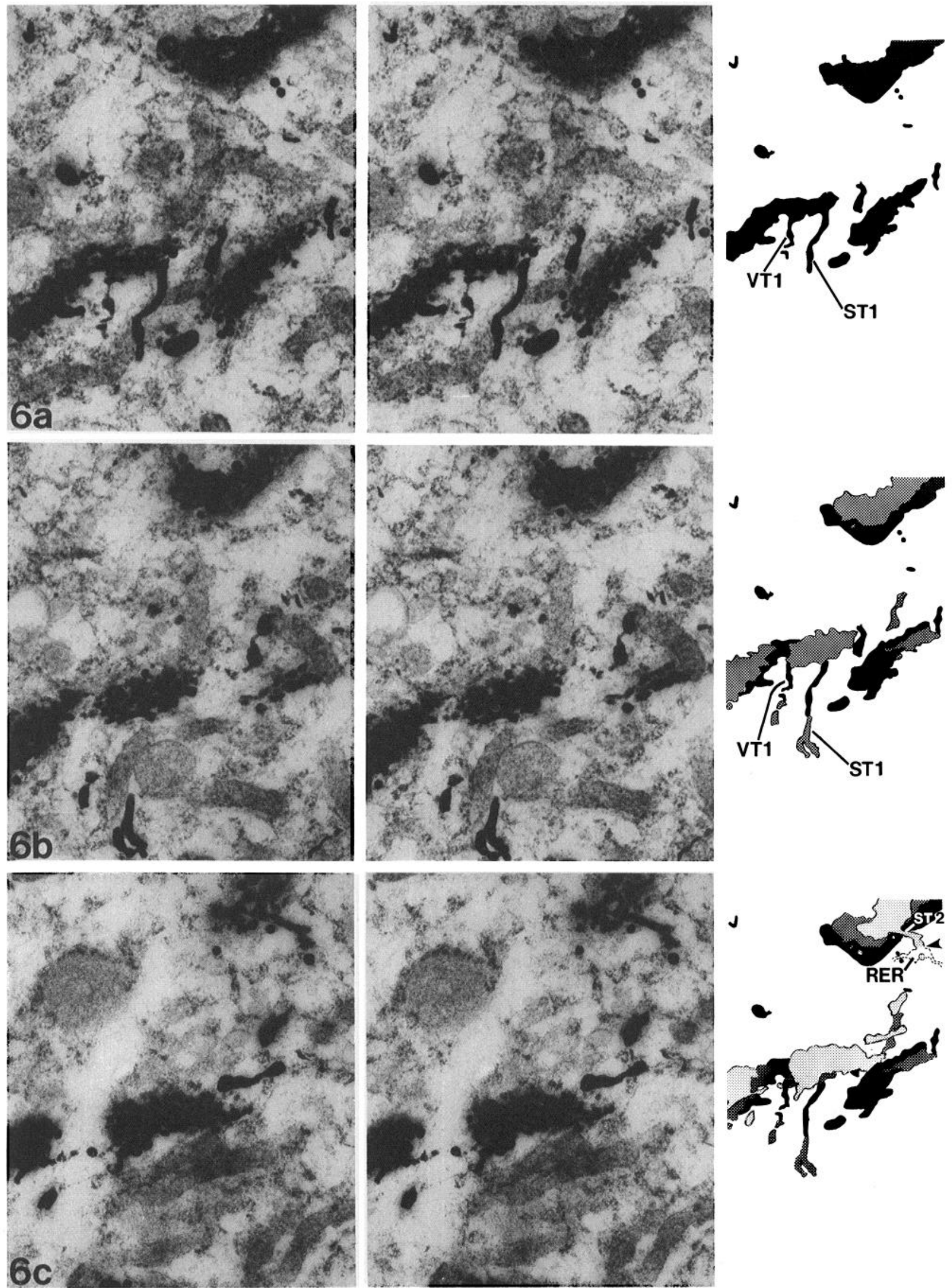

Figures 6 to 9. Serial reconstructions of varicose and smooth tubules illustrating their relationships to the Golgi apparatus and to RER. The tissue here, more heavily osmium impregnated than the examples shown previously, was stained en bloc with lead aspartate before embedding. All images in these figures are from sections $170 \mathrm{~nm}$ thick viewed at $100 \mathrm{KeV}$. The stereo pairs are constructed so that structures that leave the front plane of the section (closest to the observer) in a enter the back of the section in $b$. This relationship is maintained for the successive panels of the stereo-serial series. The drawings accompanying each stereo pair of the serial series reconstruct the three-dimensional relationships of impregnated structures cut apart during sectioning. Their alignments have been verified using the low magnification transparency overlays. The profiles in each drawing are shade coded as follows: the darkest shade, profiles from $a$; medium shading, from $b$; light shading, from $c$; no shading, from $d$. 

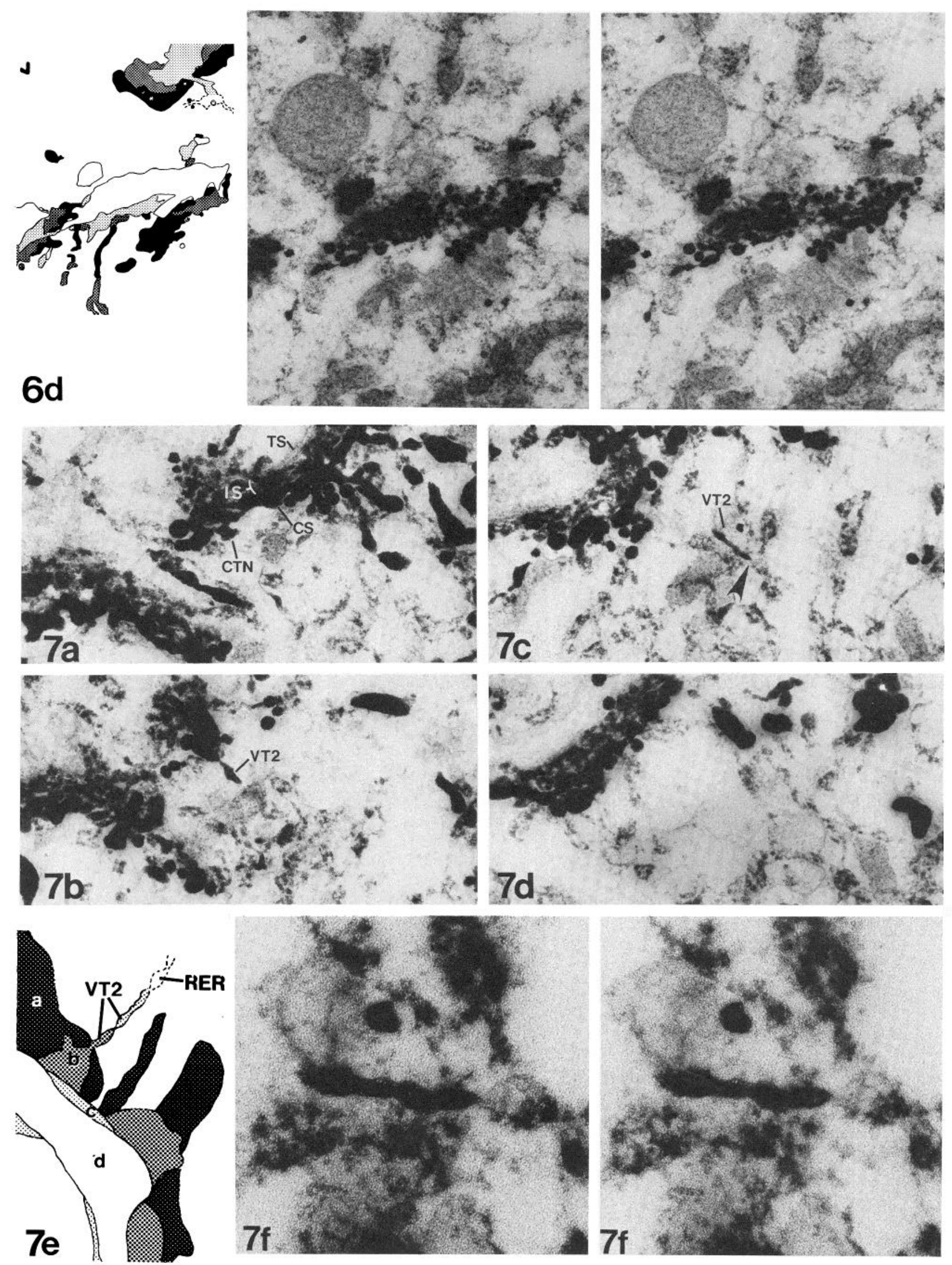

Figure 6. Serial-stereo series of a varicose tubule and two smooth tubules which arise from the cis element of the Golgi apparatus. The varicose tubule (VII) arises from the cis element and terminates blindly within a few impregnated small cisternae nearly in b. ST1, a smooth tubule, clearly terminates blindly within the middle of the section seen in stereo pair b. Like VT1, several discrete impregnated cisternae cluster near the ending of ST1. ST2 arises from the edge of the cis element and, after a short distance, connects with RER within $c$. Examination of $b$ and $d$ confirm that the origin and ending of ST2 are totally contained within the section shown in c. a to $d, 14^{\circ}$ tilt. Magnification $\times 33,000$.

Figure 7. a to $d$ comprise a serial series showing a varicose tubule arising from a network-saccule cis element (VT2 in b) and connecting to an RER element (arrowhead in $c$ ). $a$ and $d$ illustrate that VT2 is totally contained in $b$ and $c$. This series is diagrammatically reconstructed in $e$. $d$ confirms stereoscopically the transition from varicose tubule to RER shown above in $c$. To aid orientation, the following structures are also labeled: the unimpregnated trans saccule (TS), impregnated intermediate saccules (IS), and segments of the cis tubular network (CTN). CS, cis saccule. Magnifications: a to $d, \times$ $48,000 . c,: 10^{\circ}$ tilt, $\times 65,000$. 

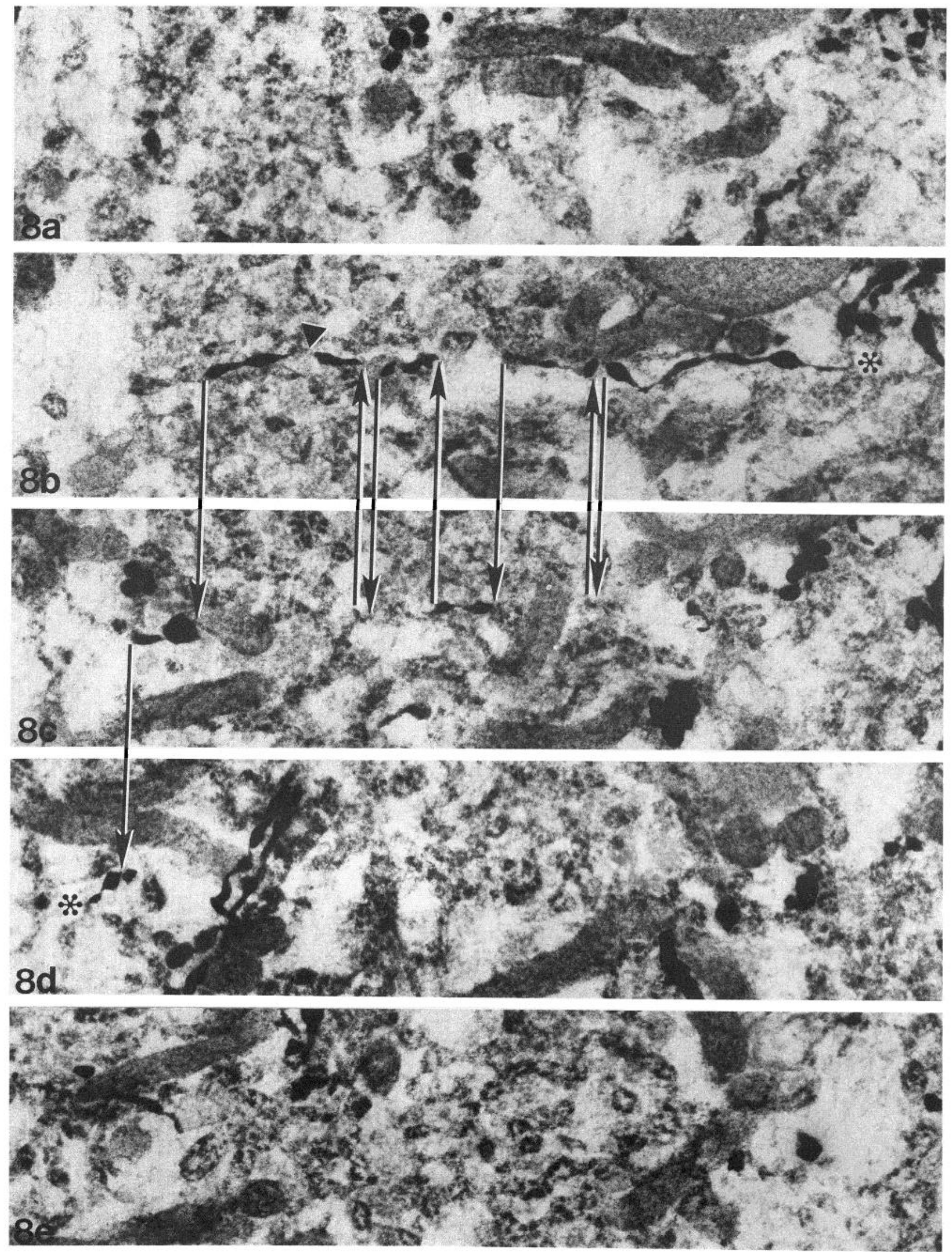

Figure 8, a-e 


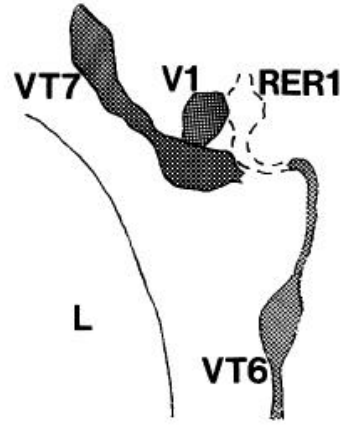

8b'
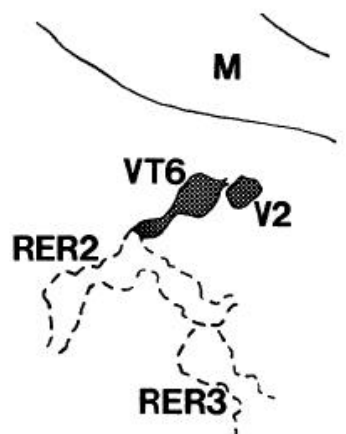

$8 d^{\prime}$
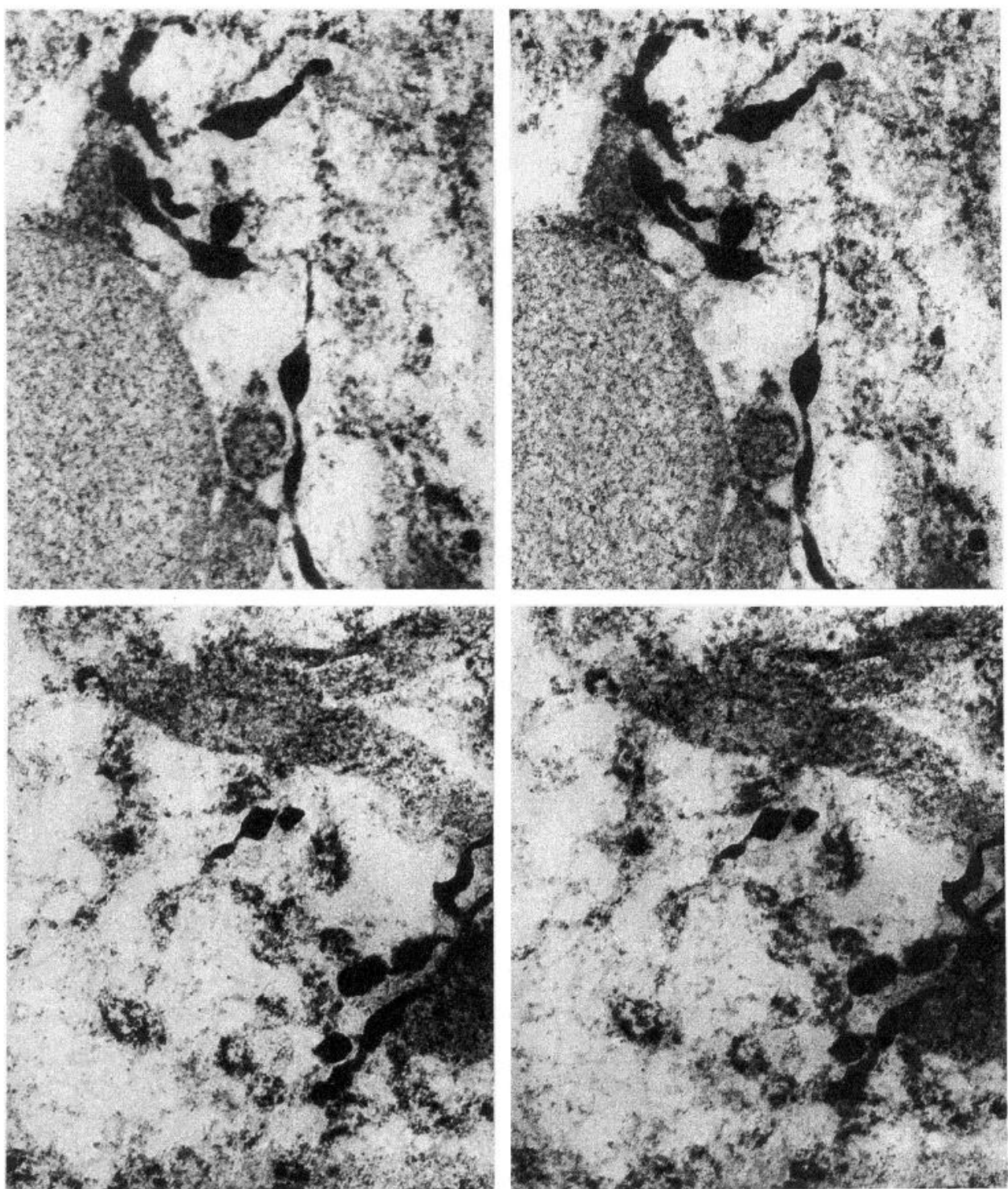

Figure 8. Serial series of a varicose tubule (VT6) that is connected on both ends to RER. One end anastomoses with RER on the right side of $b$ (asterisk). As it progresses, it passes back and forth between $b$ and $c$ as indicated by the arrows. In one place, it is grazed at the surface of the section but still remains partially within $b$ (arrowhead). Finally, it dips to the section shown in $d$ where it connects with RER (asterisk). $a$ and $e$ are included to show that VT6 is totally contained within $b, c$, and $d$. High magnification stereo pairs of the two ends of the tubules are shown in $b^{\prime}$ and $d^{\prime}$. In $b^{\prime}, V T 6$ is seen to transform to an RER element (RER1) that passes partially in front of a second varicose tubule ( $(V T 7)$ and then partially behind a vesicle $(V 1)$ before exiting the section. In panel $d^{\prime}$, the other end of VT6 transforms to an RER element (RER2) that passes in front of another RER element (RER3) before leaving the section. $L$, Iysozome; $M$, mitochondrion. Magnifications: $a$ to $e, \times 48,000 . b^{\prime}$ and $d^{\prime}, 10^{\circ}$ tilt, $\times 75,000$.

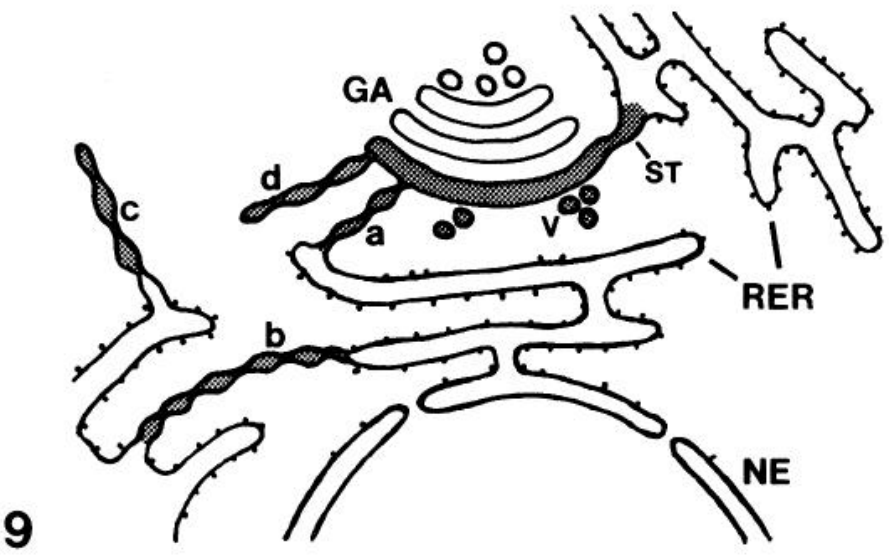

Figure 9. Diagram summarizing endomembrane relationships among RER elements and the Golgi apparatus. Varicose tubules establish direct links between RER and the cis element of the Golgi apparatus $(G A, a)$ as well as direct links between separated elements of RER $(b)$. Varicose tubules also extend out from RER (c) and from the cis element $(d)$ to form blind endings. Smooth tubules also linked RER to the element (ST) and extended from the cis element to form blind endings as well (not shown). Osmiophillic vesicles seen near the cis element $(V)$ are ideally positioned to deliver material to or from the cis element. $N E$, norepinephrine. 


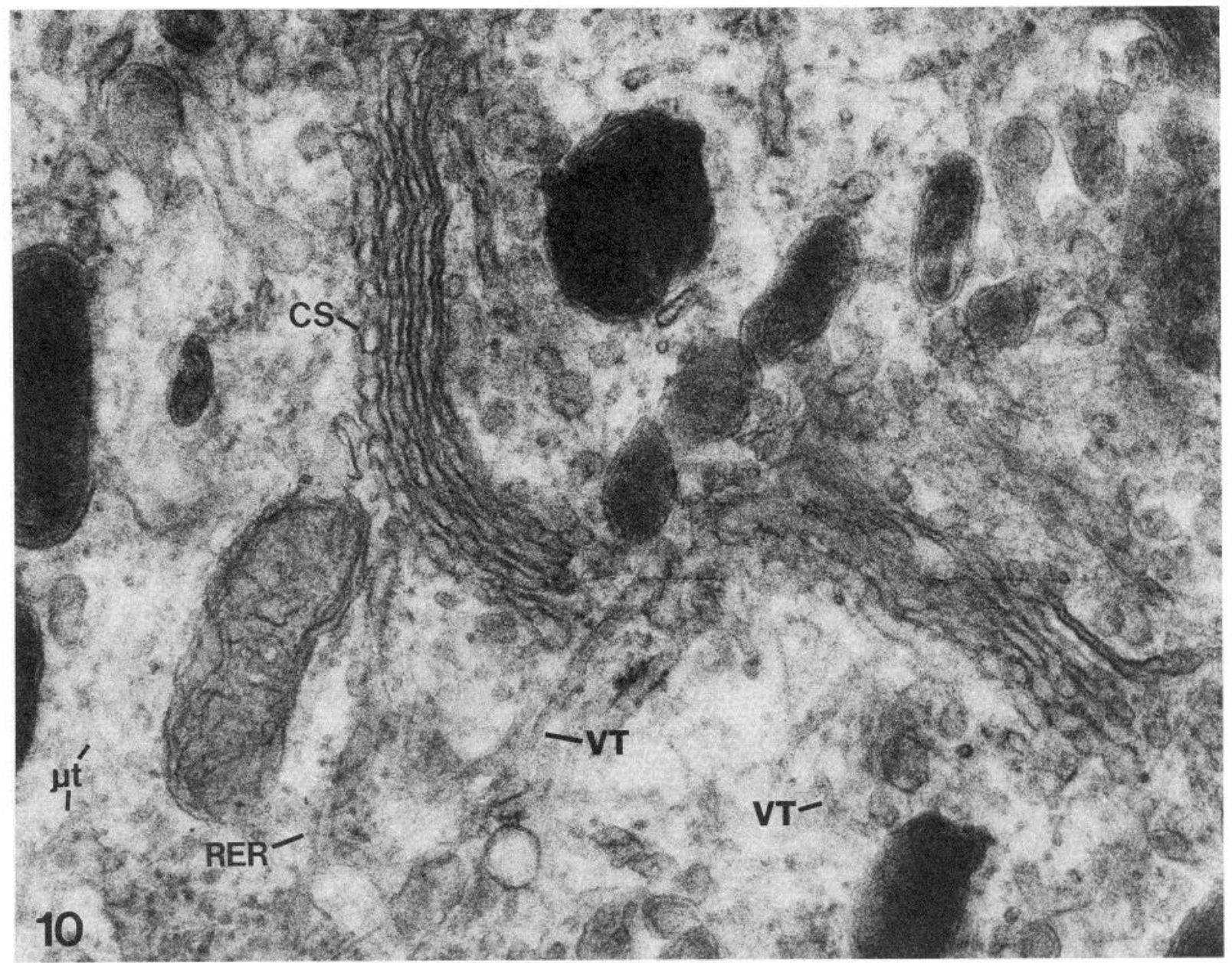

Figure 10. Re-examination of the lower half of the conventionally prepared thin section image of the Golgi apparatus shown in Figure $1 \mathrm{~b}$ to illustrate the differentiation of microtubule $(\mu t)$ and varicose tubules $(V T)$. These structures are similar in size and appearance and thus may be easily confused. The most definitive difference is that varicose tubules have unit membranes whereas microtubules do not. This unit membrane can be seen in the varicose tubule shown in the lower right portion of the figure. A more practical difference for sections 70 to $90 \mathrm{~nm}$ thick is that varicose tubules stain more darkly with lead than do microtubules. Also, varicose tubules are often somewhat kinked whereas microtubules are usually straight or gently curving. Other labeled structures include: the cis saccule of the Golgi apparatus $(C S)$ and RER elements (RER). Magnification $\times 67,000$.

$200 \mathrm{~nm}$ due to the confusing overlap of electron-dense structures. A similar relative hierarchy of osmium impregnability has been reported for plant cell organelle systems (Poux, 1973).

\section{References}

Bergmann, J. E., and S. J. Singer (1983) Immunoelectron microscopic studies of the intracellular transport of the membrane glycoprotein $(G)$ of vesicular stomatitis virus in infected Chinese hamster ovary cells. J. Cell Biol. 97: 1777-1787.

Broadwell, R. D., and A. M. Cataldo (1983) The neuronal endoplasmic reticulum: Its cytochemistry and contribution to the endomembrane system. I. Cell bodies and dendrites. J. Histochem. Cytochem. 31: 10771088.

Carasso, N., L. Ovtracht, and P. Favard (1971) Observations en microscopie électronique haute tension, de l'appareil de Golgi sur coupes de 0,5 á $5 \mu$ dépaisseur. Comp. Rend. Hebd. Ser. D. 273: 876-879.

Claude, A. (1970) Growth and differentiation of the cytoplasmic membranes in the course of lipoprotein granule synthesis in the hepatic cell. I. Elaboration of elements of the Golgi complex. J. Cell Biol. 47: 745-766.

Droz, B. (1967) Synthèse et transeret des proteines cellulaires dans les neurones ganglionnaires. Étude radioautographique quantitative en microscopie èlectronique. J. de Microscopie 6: 201-228.

Droz, B., and H. L. Koenig (1970) Localization of protein metabolism in neurons. In Protein Metabolism of the Nervous System, A. Lajtha, ed., pp. 93-108, Plenum Press, New York.
Ellisman, M. H. and J. D. Lindsey (1982) Organization of axoplasm: Mem braneous and fibrillar components possibly involved in fast neuroplasmic transport. In Axoplasmic Transport, D. Weiss, ed., pp. 55-63, SpringerVerlag, New York.

Farquhar, M. G. and G. E. Palade (1981) The Golgi apparatus (complex)(1954-1981)-From artifact to center stage. J. Cell Biol. 91: 77s-103s.

Flickinger, C. J. (1969) The development of Golgi complexes and their dependence upon the nucleus in amebae. J. Cell Biol. 49: 250-262.

Franke, W. W., and J. Kartenbeck (1976) Some principles of membrane differentiation. In Progress in Differentiation Research, N. Mueller-Berat, ed., pp. 213-243, Elsevier-North Holland Publishing Co., Amsterdam.

Grafstein, B., and D. S. Forman (1980) Intracellular transport in neurons. Physiol. Res. 14: 1167-1283.

Hammerschlag, R., G. C. Stone, J. D. Lindsey, and M. H. Ellisman (1982) Evidence that all newly synthesized proteins destined for fast axonal transport pass through the Golgi apparatus. J. Cell Biol. 93: 568-575.

Holtzman, E. (1971) Cytochemical studies of protein transport in the nervous system. Philos. Trans. R. Soc. Lond. (Biol). 261: 407-421.

Holtzman, E., and A. M. Mercurio (1980) Membrane circulation in neurons and photoreceptors. Int. Rev. Cytol. 67: 1-67.

Inferra, C., and G. Carrozza (1975) The discovery of the Golgi apparatus by the black Rect. and its present fine structural visualization. In Golgi Centennial Symposium Proceeding, M. Santini, ed., pp. 13-38, Raven Press, New York.

Jamieson, D. S., and G. E. Palade (1968a) Intracellular transport of secretory proteins in the pancreatic exocine cell. III. Dissociation of intracellular 
transport from protein synthesis. J. Cell Biol. 39: 580-588.

Jamieson, D. S., and G. E. Palade (1968b) Intracellular transport of secretory proteins in the pancreatic exocine cell. IV. Metabolic requirement. J. Cell Biol. 39: 503-522

Lindsey, J. D., and M. H. Ellisman (1983) The origin of the axoplasmic reticulum within the axon hillock. Soc. Neurosci. Abstr. 9: 1192.

Lindsey, J. D. and M. H. Ellisman (1985a) The neuronal endomembrane system. II. The multiple forms of the Golgi apparatus cis element. J. Neurosci. 5: 3124-3134.

Lindsey, J. D., and M. H. Ellisman (1985b) The neuronal endomembrane system. III. The origins of the axoplasmic reticulum and discrete axonal cisternae at the axon hillock. J. Neurosci. 5: 3135-3144.

Maul, G. G., and B. R. Brinkley (1970) Golgi apparatus during mitosis in human metanoma cells in vitro. Cancer hes. 30: $2326-2332$.

Morré, D. J. (1981) Exocytosis/flow rates and kinetics as related to membrane recycling. In International Cell Biology 1980-1981, H. Sweiger, ed., pp. 622-623, Springer-Verlag, Berlin.

Morré, D. J., and L. Ovtracht (1977) Dynamics of the Golgi apparatus: Membrane differentation and membrane flow. Int. Rev. Cytol 5: 61-188.

Novikoff, A. B., P. S. Roheim, and N. Quintana (1966) Changes in rat liver cells induced by orotic acid feeding. Lab. Invest. 15: 27-49.

Palade, G. (1975) intracellular aspects of the process of protein synthesis.
Science 189: 347-358.

Poux. N. (1973) Observation en microscopie électronique de cellules vegetales imprégnées par l'osmium. Comp. Rend. Hebd. Ser. D.276: 21632166.

Rambourg, A., and M. Chrêtien (1970) L'appareil de Golgi; examen en microscopie électronique de coupes épalsses $(0.5-1 \mu)$ aprés imprégnation de tissus par le tétroxyde d'osmium. Comp. Rend. Hebd. Ser. D270: 981983

Rambourg, A., A. Marraud, and M. Chrétien (1973) Tridimensional structure of the forming face of the Golgi apparatus as seen in the high voltage electron microscope after osmium impregnation of the small nerve cells in the semilunar ganglion of the trigeminal nerve. J. Microsc. 97: 49-57.

Rambourg, A., Y. Clermont, and A. Marraud (1974) 3-Dimensional structure of osmium impregnated Golgi apparatus as seen in high voltage electron microscope. Am. J. Anat. 140: 27-45.

Rothman, J. E. (1982) The Golgi apparatus: Roles for distinct "cis" and "trans" compartments. Ciba Found. Symp. 92: 120-137.

Tartakoff, A. M. (1980) The Golgi-complex-crossroads for vesicular traffic. Int. Rev. Exp. Pathol. 22: 227-251.

Walton, D. (1977) Lead aspartate, an en bloc contrast stain particularly useful for ultrastructural enzymology. J. Histochem. Cytochem. 27: 1337-1342. 